\title{
Selection mechanism at the onset of active turbulence
}

Berta Martínez-Prat ${ }^{1,2}$, Jordi Ignés-Mullol ${ }^{1,2}$, Jaume Casademunt ${ }^{3,4}$ and Francesc Sagués ${ }^{1,2}$

${ }^{1}$ Departament de Ciència dels Materials i Química Física, Universitat de Barcelona, 08028 Barcelona, Catalonia, Spain

${ }^{2}$ Institute of Nanoscience and Nanotechnology, Universitat de Barcelona, 08028 Barcelona, Catalonia, Spain

${ }^{3}$ Departament de Física de la Matèria Condensada, Universitat de Barcelona, 08028 Barcelona, Catalonia, Spain

${ }^{4}$ Universitat de Barcelona Institute of Complex Systems (UBICS), Universitat de Barcelona, 08028 Barcelona, Catalonia, Spain

Active turbulence describes a flow regime that is erratic, and yet endowed with a characteristic length scale ${ }^{1}$. It arises in animate soft-matter systems as diverse as bacterial baths ${ }^{2}$, cell tissues ${ }^{3}$ and reconstituted cytoskeletal preparations ${ }^{4}$. However, the way that these turbulent dynamics emerge in active systems has so far evaded experimental scrutiny. Here we unveil a direct route to active nematic turbulence by demonstrating that, for radially aligned unconfined textures, the characteristic length scale emerges at the early stages of the instability. We resolve two-dimensional distortions of a microtubule-based extensile system ${ }^{5}$ in space and time, and show that they can be characterized in terms of a growth rate that exhibits quadratic dependence on a dominant wavenumber. This wavelength selection mechanism is justified on the basis of a continuum model for an active nematic including viscous coupling to the adjacent fluid phase. Our findings are in line with the classical pattern-formation 
studies in non-active systems ${ }^{6}$, bettering our understanding of the principles of active selforganization, and providing potential perspectives for the control of biological fluids.

Active materials constitute a fascinating arena for the study of self-organization under nonequilibrium conditions ${ }^{7-9}$. Somehow surprisingly, progress in this field has gone rather unaware of the body of knowledge that has accumulated in the past around the concept of pattern forming instabilities in driven non-equilibrium systems ${ }^{6}$. Distinctively, patterns and accompanying flows in active systems emerge at time and length scales that are neither dictated by any external intervention nor by any preexisting layout. One of the most remarkable signatures of such self-sustained behaviours is the development of what has been known as active turbulence ${ }^{10-12}$. This term refers to collectively organized large-scale flows of discrete active units of very different nature ${ }^{2-4}$. These flows, commonly pervaded by a significant number of topological defects, evolve apparently deprived of any spatio-temporal coherence. Notwithstanding, they organize around an active length scale, defined as a ratio of elasticity and activity, that sets a typical separation distance between defects in the fully developed turbulent regime ${ }^{1,4,13,14}$. How this length scale emerges, or whether it may arise from a linear or a non-linear mechanism is not clear.

Ideally aligned active nematics are prone to destabilize under extensile or contractile stresses to give rise, respectively, to bend or splay long-range distortions. Such a reorientational instability has been known for years ${ }^{15-17}$, though it has never been portrayed at onset with specifically dedicated experiments. By onset we refer hereafter to the early stage development of the unconstrained dynamics in the active sample. The closest reference is the recently published work by Duclos et 
al. reporting spontaneous flows for spindle-shape cells confined in stripes ${ }^{18}$. However, these flows do not exhibit an intrinsic length scale, but are system-size dependent, and thus they are not enforced by a genuine wavelength selection mechanism. Here we demonstrate from experiments with microtubule-based active nematics and active-gels theory, that the length scale at fully developed turbulence is selected right at the onset of the initial reorientational instability of a radially-aligned active nematic free of confinement. In our experiments, the selection mechanism is understood in terms of a balance between activity, as a destabilizing force acting over the whole wavenumber range, and a pair of stabilizing effects playing distinctively at both ends of the spectrum: at the largest wavenumbers, stabilization is associated to the nematic elasticity of the material while, at the smallest range, both a geometric effect of the radial symmetry, and the hydrodynamic coupling to the adjacent viscous fluids, suppress the long wave-length instability.

The chosen material is based on the self-assembly of micrometer-sized stabilized microtubules (MTs) condensed at an oil/water interface from a bulk active-gel preparation ${ }^{4,5,19}$ (see Methods and Fig. S1). Microtubules are bundled under the depleting action of polyethilene-glycol (PEG). This facilitates cross-linking and internal shearing by kinesin motors clustered with streptavidin (See Methods). Fueled with adenosine triphosphate (ATP), bundles are permanently subjected to extension and buckling. Left to evolve free of constrainments, the two-dimensional active layer adopts a characteristic long range orientational (nematic) order, continuously permeated by large-scale flows and locally interrupted by regions void of MTs that configure semi-integer defects. The above scenario, displaying a permanently renovated dynamics of defect creation and annihilation ${ }^{20-22}$, corresponds to the state of active nematic turbulence, whose origin we unveil 
here.

We begin by aligning the active nematic in contact with a glass tube vertically placed at the oil/water interface (see Fig.S1). In-flow currents flowing into the tube align the nematic active sample with bundles laying radially around the area where the tube is positioned (see Fig. 1a). After removal of the capillary, this preparation is unstable, being immediately disrupted by the spontaneous buckling of the aligned extensile material, leading to the development of a bendtype distortion (see Fig. 1b). The instability organizes a pattern of periodically-spaced concentric crimps that evolve into circular walls. Elastic stress accumulated in the walls is released through the nucleation of pairs of $\pm 1 / 2$ defects ${ }^{13}$ that align along dark circular lanes (Fig. 1c). Subsequent defect motion dismantles the circular pattern (see Fig. 1d), and reverts the system into a turbulent regime (see Video S1).

The development of the fully turbulent regime is better appraised in situations of low motor concentration (see Video S2). In these cases, the instability repeats periodically following orthogonal directions until the material arrives at the characteristic turbulent state. First, self-propelled defects remain confined within the circular lanes originated in the original bend instability. Defect motion exerts a shear flow that forces the progressive tangential alignment of the bundles between neighbouring lanes. In this aligned configuration, the system is again prone to the bend instability, which this time organizes radially-directed walls, along which new motile defects unbundle and propel (see Fig. 2a-d). As the wavelength of the instability keeps practically unaltered, the signature of the whole process is illustrated by lines of moving defects that compose a square grid after 
time averaging the recorded fluorescence micrographs (see Fig. 2e). Alternation between radial and azimuthal modes is evidenced by the trace of the corresponding FFT amplitudes (See Fig. 2f).

This pattern forming instability can be quantitatively accounted in terms of the characteristic wavenumber, $k^{*}$, and growth rate, $\Omega^{*}$ corresponding to the leading (most unstable) mode of distortion (see Methods and Figs. S2 and S3). Four different control parameters were considered, i.e. ATP, motor units, microtubules and PEG. ATP concentration is considered a direct measure of the chemical energy fueled into the system, while the latter three are presumably related both to the power effectively transduced on the microtubule network, and to its mechanical properties.

The first set of results corresponds to varying the ATP concentration. Plots of both $k^{*}$ and $\Omega^{*}$ indicate that the instability is stronger (i.e. smaller imprinted wavelength, or, equivalently, larger wavenumber, and faster response) when increasing ATP concentration (see Fig. 3a). Experiments with MT-based active nematics have shown that the activity, $\alpha$, is related to a logarithmic measure of the concentration of ATP ${ }^{19}$. Therefore, we will use $\alpha \simeq \ln [A T P]$ as the experimental activity in our analysis, assuming all other control parameters are kept constant. For very low $\alpha$, the extracted $k^{*}$-values collapse into a plateau, as the corresponding wavelengths become of the order of the sample size (a few hundreds of microns). Past a threshold activity, we find that our data are compatible with both $\left(k^{*}\right)^{2}$ and $\Omega^{*}$ increasing linearly with $\alpha$. The role of motor units (see Fig. 3b) is put into evidence by the direct linear dependence of both $k^{*}$ and $\left(\Omega^{*}\right)^{1 / 2}$ when increasing the concentration of clustered kinesins. The dependence on microtubules contents shows that $k^{*}$ and $\Omega^{*}$ decrease past a range of minimal microtubule concentration (see Fig. 3c). Finally, a non- 
monotonous behaviour is observed when varying the concentration of the PEG depleting agent (see Fig. 3d). At small concentrations, an increase in [PEG] renders the system unstable towards smaller wavelengths. Conversely, high concentrations of PEG render the material more rigid, and it is only able to accommodate distortions of wavelength comparable to the system size.

Interestingly, the separated datasets collapse into a single master curve, $\Omega^{*} \sim\left(k^{*}\right)^{2}$ (see Fig. 3e), that fully characterizes the involved length and time scales at the onset of the bending instability of the active nematic.

Wavelength selection is rationalized within the theory of active gels ${ }^{23,24}$. We build upon the analysis by Voituriez et al. ${ }^{25}$ for parallel alignment. In an unconfined system and for extensile stresses, this gives a linear growth rate of the form $\Omega(k)=a^{\prime}-b^{\prime} k^{2}$, for longitudinal perturbations (see S.I.), which yields no intrinsic wavelength selection. In the case of radial alignment, the growth rate spectrum is expected to be discrete and hence necessarily select a finite scale with maximal growth rate. However, the effect of the unavoidable viscous coupling to liquids in contact with the active nematic fluid ${ }^{26}$ has been shown to qualitatively modify the growth rate for parallel alignment into the form $\Omega(k)=a k-b k^{2},{ }^{27,28}$ which indeed provides a genuine selection mechanism at the maximal growth rate. Therefore, for the benefit of discussion and physical insight, we will assume the latter form of growth rate as an appropriate qualitative description valid also for radial case.

The parameter $a$ of the linear part in the dispersion relation just quoted should depend on activity, but in a way that is not known precisely, while $b$, characterizing the equilibrium elastic 
relaxation, is explicitly known in terms of the material parameters (see SI). A critical wavenumber $k_{c}=a / b$, above which random perturbations decay, sets the upper bound on the instability range, while the maximum growth rate is set at $k^{*}=k_{c} / 2$, independent of material parameters. Accordingly, we may assume that, for the real system at hand, we will generically have $k^{*}=\theta k_{c}$ with $\theta<1$ a numeric factor to be determined but essentially independent from parameters. From the experimental measurements of $k^{*}$ we may estimate $a$ from the relationship $a=b k_{c}$, however, since the value of $k_{c}$ should approach the one in the absence of viscous coupling in the appropriate limit we may expect $a \propto(\alpha)^{1 / 2}$ (see $\mathrm{SI}$ ).

Interestingly, the growth rate of the selected mode $\Omega^{*}=\Omega\left(k^{*}\right)$ can be written in the form $\Omega^{*}=b\left(k^{*}\right)^{2}$, where $k^{*} \propto(\alpha / K)^{1 / 2}$ (see S.I.). Notice that this is the well-known scaling reported in the literature for the active length scale. Using the explicit form of $b$ (see SI), this results in

$$
\Omega^{*}=\frac{K}{\gamma}\left(1+\frac{\gamma}{4 \eta}(1-\nu)^{2}\right)\left(k^{*}\right)^{2} .
$$

From the above considerations, we readily interpret the experimental results when varying ATP contents (i.e. $k^{*} \propto \alpha^{1 / 2}$ ). On what respects to kinesin concentration, the experimental scaling can be understood by referring to earlier studies on the velocity of the active microtubule-based material. First, experiments ${ }^{29}$ suggest that this velocity and motor concentration are linearly related in this concentration range. On the other hand, the scaling $v \propto \alpha^{1 / 2}$ has been predicted theoretically and confirmed experimentally ${ }^{1,19}$. Consequently, the linear scaling of $k^{*}$ with motor concentration (see Fig. 3b) is fully consistent. Concerning MTs contents, the behaviour (see Fig. 3c) can be interpreted assuming, plausibly, that when densifying the active nematic by in- 
creasing the MT concentration, the system becomes stiffer (larger $K$ ), making $\mathrm{k}^{*}$ and $\Omega^{*}$ smaller. Finally, we can now interpret the two regimes observed when changing the concentration of PEG. At low concentrations, PEG makes the system more efficient by optimizing the motor translocation, thus resulting in an enhanced effective $\alpha$. At high PEG concentrations, our results highlight that beyond a threshold, PEG has a similar effect to the one observed for the case of increasing MTs concentration.

One of the main results of this analysis is Eq.(1), which justifies the observed scaling with a constant of proportionality that depends solely on material parameters $(K, \gamma, \eta, \nu)$ but not on the activity. In this way, we conclude that there is a characteristic time scale of the emerging pattern as well, and it is given as a simple ratio of the activity and shear viscosity parameters (see SI), $\Omega^{*} \propto \frac{\alpha}{\eta}$. We identify this time scale as characteristic of the turbulent regime, as previously suggested ${ }^{30}$.

The presented results demonstrate a route to active turbulence in nematics endowed with characteristic length and time scales that are imprinted right at the onset of an extensional instability of the material. These results should motivate a renovated interest towards a better understanding of the fundamentals and realizations of active flows, both in laboratory and natural systems. 

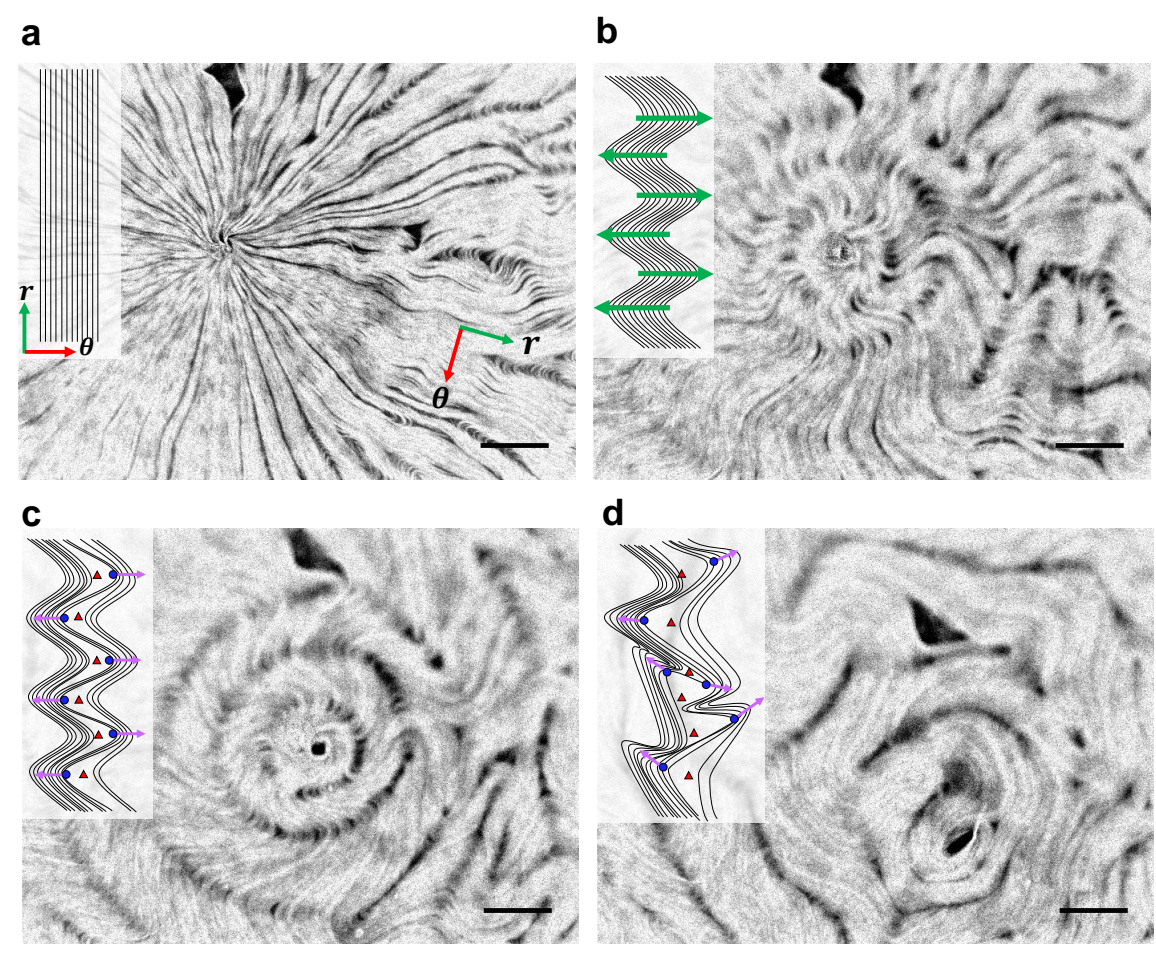

Figure 1 Route to active turbulence. Fluorescence micrographs showing the spontaneous evolution of a radially aligned active nematic towards the turbulent regime. A diagram illustrating the disposition of MT bundles is included as an inset with each frame. Elapsed times from frame a are $13 \mathrm{~s}$ (b), $26 \mathrm{~s}$ (c) and $47 \mathrm{~s}$ (d). a Radially-aligned AN. The polar coordinate variables are sketched on the image and in the diagram. b Onset of the bending instability. The green arrows in the diagram indicate the active stresses that lead to the formation of crimps. $c$ Nucleation of $\pm 1 / 2$ defect pairs within the crimps, leading to a degradation of the orientational order. In the diagram, $-1 / 2$ defects are the red triangles and $+1 / 2$ are the blue circles. Because of their geometry, $+1 / 2$ defects can self-propel and travel transiently along the crimps. d Eventually, $+1 / 2$ defects alter their trajectories and finally break the patterned-structure. Scale bars $=100 \mu \mathrm{m}$. Experimental conditions are [ATP] $=280 \mu \mathrm{M}$, [Streptavidin] $=8.2 \mu \mathrm{g} / \mathrm{mL}$, [MTs] $=1.3 \mathrm{mg} / \mathrm{mL}$ and [PEG]=1.7\%(w/w). 

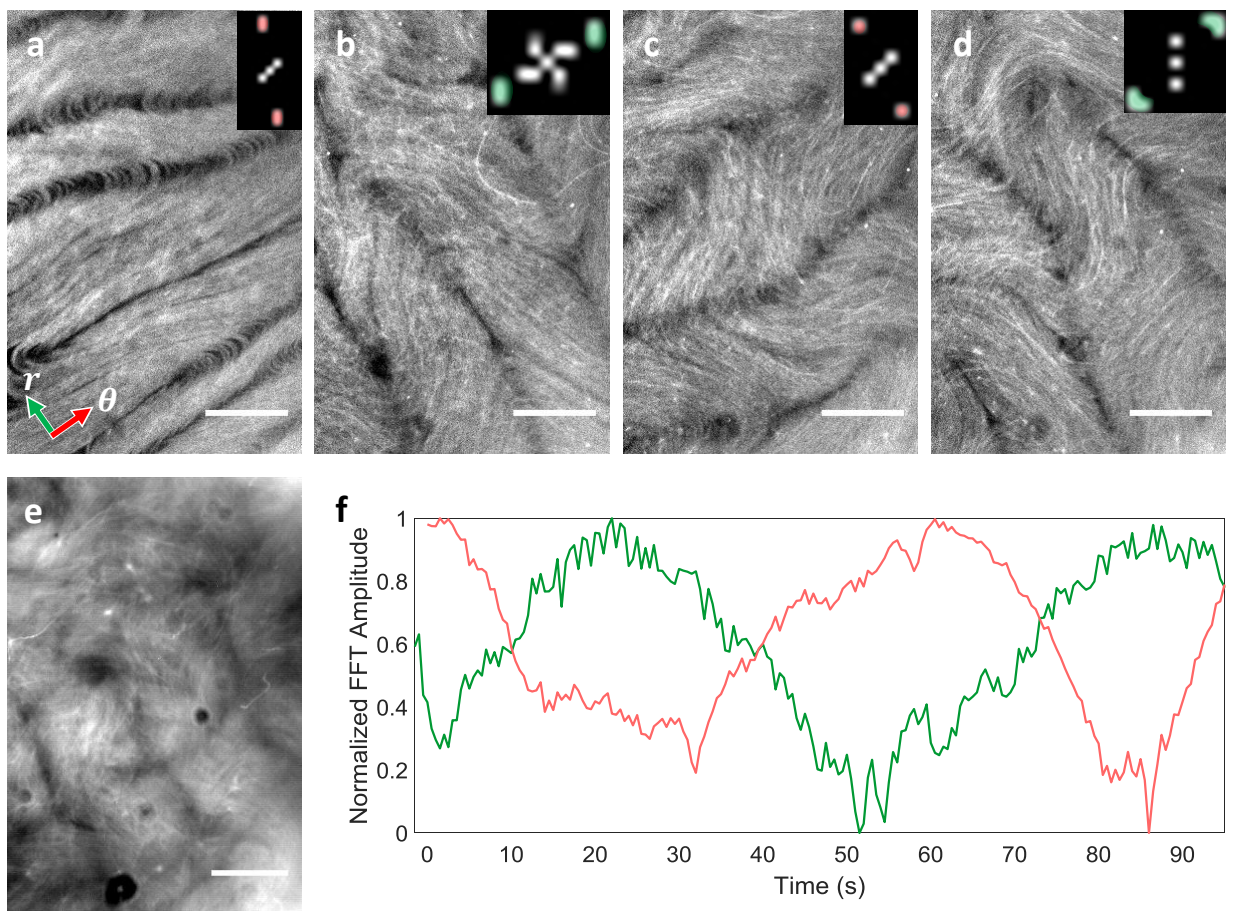

Figure 2 Sequential instabilities. a-d Fluorescence micrographs displaying patterns formed by consecutive instabilities that evolve in orthogonal directions. The polar coordinate variables are sketched in a. Elapsed times from (a) are $22 \mathrm{~s}$ (b), $63 \mathrm{~s}$ (c), and $92 \mathrm{~s}$ (d). The amplitude of the 2D FFT is included as an inset for each image. For clarity, we have highlighted in the FFTs the peaks corresponding to every pattern emerged at the azimuthal and radial directions in red and green, respectively. e Time average of all the micrographs from (a) to (d) evidencing the orthogonal arrangement of the dark lanes in fluorescence micrographs. f Normalized FFT amplitude in the peaks corresponding to patterns formed along the radial direction (green trace) and along the azimuthal direction (red trace). Scale bars $=100 \mu \mathrm{m}$. Experimental conditions are [ATP]=1.5 mM, [Streptavidin] $=7.5 \mu \mathrm{g} / \mathrm{mL},[\mathrm{MTs}]=1.3 \mathrm{mg} / \mathrm{mL}$ and $[\mathrm{PEG}]=1.7 \%(\mathrm{w} / \mathrm{w})$. 

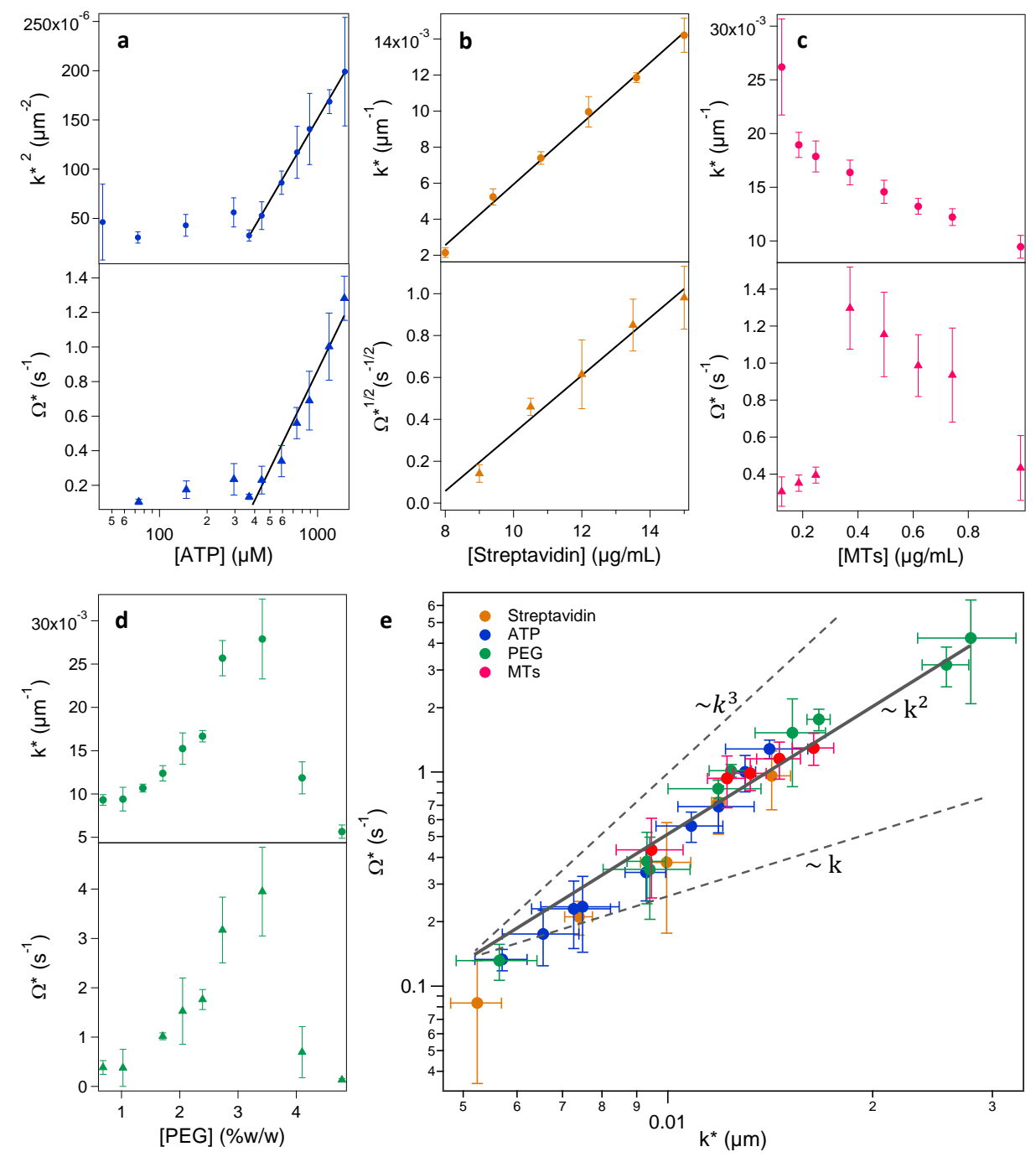

Figure 3 Dependence of $\mathbf{k}^{*}$ and $\Omega^{*}$ with material parameters and scaling $\Omega^{\star}\left(\mathbf{k}^{\star}\right)$ a-d Experi-

mental scaling relations of the characteristic wavenumber, $k^{*}$, and growth rate, $\Omega^{*}$, of the instability of aligned active nematics, as a function of the different control concentrations. In (a) and (b) solid lines are guides for the eye in regions where scaling is observed. e $\Omega^{*} v s . k^{*}$ for all experiments. Data collapse in a single master curve that can be well fitted by $\Omega^{*} \propto k^{* 2}$ (solid line). For comparison, other power law relations are included as dashed lines. Error bars in $k^{*}$ are the standard 
deviation for measurements of different images. Error bars in $\Omega^{*}$ are the uncertainties from the data fitting (see Fig. S3(e)). 


\section{Methods}

MTs polymerization $\sim 1 \mu \mathrm{m}$ MTs were polymerized using heterodimeric $(\alpha, \beta)$-tubulin from bovine brain (from the Brandeis University Biological Materials Facility (Waltham, MA)). The polymerization was carried out in an aqueous solution of M2B buffer ( $80 \mathrm{mM}$ Pipes (piperazineN,N-bis(2-ethanesulfonic acid)), 1mM EGTA (ethylene glycol-bis(-aminoethyl ether)-N,N,N',N'tetraacetic acid), $2 \mathrm{mM} \mathrm{MgCl}_{2}$ and Mili-Q water) (Sigma; P1851, E3889 and M4880, respectively) with a total tubulin concentration of $8 \mathrm{mg} / \mathrm{ml}$, of which a $3 \%$ had been labeled with Alexa 647 to enable the observation under fluorescence microscopy. The antioxidant agent DTT (dithiothrethiol) (Sigma 43815) and the non-hydrolysable GTP (Guansine triphosphate) analogue GMPCPP (guanosine-5' -[( $\alpha, \beta)$-methyleno]triphosphate) (Jena Biosciences, NU-405) were also added to the polymerizing mixture up to a concentration of $1 \mathrm{mM}$ and $0.6 \mathrm{mM}$, respectively. The suspension was incubated for $30 \mathrm{~min}$ at $37^{\circ} \mathrm{C}$ and afterwards annealed at room temperature for 5 hours. Finally, the solution containing the polymerized tubulin was kept at $-80^{\circ} \mathrm{C}$ until its posterior use.

Kinesin expression The kinesin used in the experiments was the heavy-chain kinesin-1 K401BCCP-6His from Drosophila Melanogaster (truncated at residue 401, fused to biotin carboxyl carrier protein (BCCP), and labeled with six histidine tags). It was expressed by Escherichia Coli using the plasmid WC2 from the Gelles Laboratory (Brandeis University) and then purified with a nickel column. Finally, the suspension was dialyzed against 500mM imidazole buffer and stored in a $40 \% \mathrm{w} / \mathrm{v}$ sucrose solution at $-80^{\circ} \mathrm{C}$. The final concentration of kinesin was estimated by means of absorption spectroscopy. 
Kinesin/streptavidin clusters Kinesin/streptavidin clusters were assembled by mixing freshly thawed K401 kinesin with streptavidin (Invitrogen 43-4301) at a ratio of 2:1 with a streptavidin concentration of $30.6 \mu \mathrm{g} / \mathrm{mL}$. DTT was added up to a concentration of $2 \mu \mathrm{M}$. The mixture was incubated on ice for $30 \mathrm{~min}$.

Assembly of the active nematic (AN) The standard preparation consisted on a M2B aqueous mixture with ATP $(1.5 \mathrm{mM})$, kinesin/streptavidin clusters $(8.2 \mu \mathrm{g} / \mathrm{mL}$ of streptavidin), MTs (1.3 $\mathrm{mg} / \mathrm{mL})$ and the depleting agent PEG $(1.7 \% \mathrm{w} / \mathrm{w})$. In order to maintain constant the concentration of ATP during the experiments, we also added an ATP-regenerating system consisting on PK/LDH (pyruvate kinase/lactate dehydrogenase) (Sigma, P-0294) (6\%vol/vol) and phosphoenol pyruvate (PEP) $(27.8 \mathrm{mM})$. The mixture also contained anti-oxidants to avoid photo-bleaching during the fluorescence imaging: DTT $(5.8 \mathrm{mM})$, glucose oxidase $(0.2 \mathrm{mg} / \mathrm{mL})$, catalase $(0.04 \mathrm{mg} / \mathrm{mL})$, trolox (6-hydroxy-2,5,7,8-tetramethylchroman-2-carboxylic acid) $(2.1 \mathrm{mM})$ and glucose $(3.5 \mathrm{mg} / \mathrm{mL})$. To have a biocompatible oil/water interface in subsequent steps, we also included in the mixture the surfactant Pluronic (Sigma P2443) (0.4\% w/v). In some cases, we varied the concentrations of ATP, motor-clusters, PEG, or MTs to study the influence of their concentration. To prepare the samples, $1 \mu \mathrm{L}$ of the final MTs suspension was introduced in a PDMS pool of $5 \mathrm{~mm}$ of diameter and $2 \mathrm{~mm}$ of depth already filled with an isotropic oil (polydimethylsiloxane (PDMS), with kinematic viscosity $1.0 \mathrm{~cm}^{2} \mathrm{~s}^{-1}$ (Rhodorsil Oils 4747 V 100 Bluestar Silicones)) (see Fig. S1). The pool was not covered, leaving the sample open. The spontaneous adsorption of the MTs onto the oil/water interface led to the formation of the active nematic. The pool had been manufactured polymerizing PDMS inside a custom-made mold and finally gluing it on a polyacrilamdide-functionalized slide 
with UV glues (Norland NOA-81).

Observation of the AN The observation of the AN layer was carried out by means of fluorescence microscopy. To this aim, we used a custom-built inverted optical microscope equipped with a white led source (Thorlabs MWWHLP1) with a Cy5 filter cube (Edmund Optics). Images were captured using a Zyla 4.2 PLUS sCMOS camera (Andor) operated with the open-source software ImageJ $\mu$-Manager.

Alignment of the AN A capillary tube (Sigma, Z611239), with outer and inner diameters of $1.2 \mathrm{~mm}$ and $0.3 \mathrm{~mm}$, respectively, was held with a linear motion stage (KDC101, Thorlabs) above the pool containing the sample and it was slowly introduced until it touched the oil/water interface, creating a radial flow that led to the alignment of the AN layer (see Fig. S1). Afterwards, the tube was carefully removed, allowing observation of the interface and enabling the AN to evolve freely.

Characterization of the bend instability wavenumber $\mathbf{k}^{*}$ A time-averaged micrograph of different frames displaying the pattern was used to measure the fluorescence intensity profile along a direction orthogonal to the crimps, using ImageJ (See Fig. S2). Finally, the wavenumber was extracted performing a FFT of the intensity profile using MatLab. The error bars were calculated as the standard deviation of 10 measurements along different radial directions in a given experimental realization.

Characterization of the bend instability time scale $\Omega^{*}$ The instability time scale was determined by monitoring the time evolution of the FFT power spectra with ImageJ. $\Omega^{*}$ was finally extracted by fitting the FFT intensity of the known $\mathrm{k}^{*}$ at short times to an exponential trend (see Fig. S3). 
Error bars correspond to the uncertainty in the fitted parameter.

1. Giomi, L. Geometry and topology of Turbulence in active nematics. Physical Review X 5, $1-11(2015)$.

2. Wensink, H. H. et al. Meso-scale turbulence in living fluids. Proceedings of the National Academy of Sciences 109, 14308-14313 (2012).

3. Blanch-Mercader, C. et al. Turbulent dynamics of epithelial cell cultures. Physical Review Letters 120, 208101 (2018).

4. Guillamat, P., Ignés-Mullol, J. \& Sagués, F. Taming active turbulence with patterned soft interfaces. Nature Communications 8, 1-8 (2017).

5. Sanchez, T., Chen, D. T. N., DeCamp, S. J., Heymann, M. \& Dogic, Z. Spontaneous motion in hierarchically assembled active matter. Nature 491, 431-434 (2012).

6. Cross, M. C. \& Hohenberg, P. C. Pattern formation outside of equilibrium. Reviews of Modern Physics 65, 851-1112 (1993).

7. Ramaswamy, S. The mechanics and statistics of active matter. Annu. Rev. Condens. Matter. Phys. 1, 323-345 (2010).

8. Marchetti, M. C. et al. Hydrodynamics of soft active matter. Reviews of Modern Physics $\mathbf{8 5}$, 1143-1189 (2013).

9. Menzel, A. M. Tuned, driven, and active soft matter. Physics Reports 554, 1-45 (2015). 
10. Bratanov, V., Jenko, F. \& Frey, E. New class of turbulence in active fluids. Proceedings of the National Academy of Sciences 112, 15048-15053 (2015).

11. Urzay, J., Doostmohammadi, A. \& Yeomans, J. M. Multi-scale statistics of turbulence motorized by active matter. Journal of Fluid Mechanics 822, 762-773 (2017).

12. Slomka, J. \& Dunkel, J. Symmetry breaking and turbulence in active fluids. Proceedings of the National Academy of Sciences 114, 15048-15053 (2017).

13. Thampi, S. P., Golestanian, R. \& Yeomans, J. M. Instabilities and topological defects in active nematics. EPL 105, 18001 (2014).

14. Giomi, L., Bowick, M. J., Mishra, P., Sknepnek, R. \& Marchetti, M. C. Defect dynamics in active nematics. Phil Trans $R$ Soc A 372, 20130365 (2014).

15. Aditi Simha, R. \& Ramaswamy, S. Hydrodynamic fluctuations and instabilities in ordered suspensions of self-propelled particles. Physical review letters 89, 058101 (2002).

16. Edwards, S. A. \& Yeomans, J. M. Spontaneous flow states in active nematics: A unified picture. Europhysics Letters 85, 18008 (2009).

17. Zhou, S., Sokolov, A., Lavrentovich, O. D. \& Aranson, I. S. Living liquid crystals. Proc Natl Acad Sci U S A 111, 1265-70 (2014).

18. Duclos, G. et al. Spontaneous shear flow in confined cellular nematics. Nat Phys 14, 728-732 (2018). 
19. Guillamat, P., Ignés-Mullol, J. \& Sagués, F. Control of active liquid crystals with a magnetic field. Proc Natl Acad Sci USA 113, 54985502 (2016).

20. Giomi, L., Bowick, M. J., Ma, X. \& Marchetti, M. C. Defect annihilation and proliferation in active Nematics. Physical Review Letters 110, 228101 (2013).

21. Thampi, S. P., Golestanian, R. \& Yeomans, J. M. Velocity correlations in an active nematic. Physical Review Letters 111, 118101 (2013).

22. Pismen, L. M. \& Sagués, F. Viscous dissipation and dynamics of defects in an active nematic interface. European Physical Journal E 40, 1-8 (2017).

23. Jülicher, F., Kruse, K., Prost, J. \& Joanny, J. F. Active behavior of the Cytoskeleton. Physics Reports 449, 3-28 (2007).

24. Prost, J., Jülicher, F. \& Joanny, J. F. Active gel physics. Nature Physics 11, 111-117 (2015).

25. Voituriez, R., Joanny, J. F. \& Prost, J. Spontaneous flow transition in active polar gels. EPL 70, 404-410 (2005).

26. Guillamat, P., Ignés-Mullol, J., Shankar, S., Marchetti, M. C. \& Sagues, F. Probing the shear viscosity of an active nematic film. Phys Rev E 94, 060602(R) (2016).

27. Gao, T., Blackwell, R., Glaser, M. A., Betterton, M. D. \& Shelley, M. J. Multiscale polar theory of microtubule and motor-protein assemblies. Physical Review Letters 114, 048101 (2015). 
28. Gao, T., Betterton, M. D., Jhang, A.-S. \& Shelley, M. J. Analytical structure, dynamics, and coarse graining of a kinetic model of an active fluid. Physical Review Fluids 2, 093302 (2017).

29. Henkin, G., DeCamp, S. J., Chen, D. T. N., Sanchez, T. \& Dogic, Z. Tunable dynamics of microtubule-based active isotropic gels. Phil Trans $R$ Soc A 372, 20140142 (2014).

30. Hemingway, E. J., Mishra, P., Marchetti, M. C. \& Fielding, S. M. Correlation lengths in hydrodynamic models of active nematics. Soft Matter 12, 7943-7952 (2016).

Acknowledgements The authors thank R. Alert and M. Shelley for fruitful discussions, and the two anonymous reviewers for their useful comments that helped improve the theoretical analysis. B.M.-P. thanks S. Marco for advising in the image analysis. The authors are indebted to the Brandeis University MRSEC Biosynthesis facility for providing the tubulin. We thank M. Pons, A. LeRoux, and G. Iruela (Universitat de Barcelona) for their assistance in the expression of motor proteins. B.M.-P., J.I.-M., and F.S. acknowledge funding from MINECO (project FIS2016-78507-C2-1-P, AEI/FEDER, EU). J.C. acknowledges support from MINECO (project FIS2016-78507-C2-2-P, AEI/FEDER, EU) and Generalitat de Catalunya under project 2014-SGR-878. B.M. acknowledges funding from UAM under the IFIMAC Master Grant, and from Generalitat de Catalunya through a FI-2018 PhD Fellowship. Brandeis University MRSEC Biosynthesis facility is supported by NSF MRSEC DMR-1420382.

Author contribution F.S., J.I.-M., and B.M-P. conceived the experiments. J.I.-M. and B.M.-P. designed the experimental setup. B.M.-P. performed the experiments. F.S., J.I.-M., and B.M.-P. analyzed and interpreted the data. J.C. performed the theoretical analysis. F.S. wrote the manuscript with collaboration from all the authors. 
Competing Interests The authors declare that they have no competing financial interests.

Data availability The data that support the plots within this paper and other findings of this study are available from the corresponding author on request. 